\title{
Structural concept, static and dynamic properties of RPC-BSS with high durability
}

\author{
L. C. Jiang ${ }^{1}$, R. Gao ${ }^{1} \&$ L. $\mathrm{Li}^{2}$ \\ ${ }^{1}$ School of Civil Engineering, Beijing Jiaotong University, P.R. China \\ ${ }^{2}$ Central Research Institute of Building and Construction, NCC Group, \\ P.R. China
}

\begin{abstract}
A new kind of beam string structure called RPC-BSS, which consists of reactive powder concrete upper chord and cables, is presented in this paper. The material of RPC provides superior mechanical properties and high durability, and the cable provides huge tensile force. The concept is based on the combination of the advantages of RPC and beam string technology. Furthermore, static and dynamic properties are discussed, and the influence of different factors is taken into consideration in the analysis. The analytical results show RPC-BSS is of longer span, less weight, better seismic characteristics and higher durability than conventional BSS.
\end{abstract}

Keywords: RPC-BSS, reactive powder concrete, high durability.

\section{Introduction}

Beam string structure (BSS) [1] is a kind of composite structure which is composed of a rigid member as the upper chord, a flexible member as the lower chord and some bracing bars as link. There are three familiar types of BSS. The first is tension beam, where the rigid member is a beam. The second called a tension arch, which is made up of a rigid arch as the upper chord. The third substitutes a two-way sloped beam for the arch.

The new material of RPC provides superior mechanical properties and high durability. The mechanical properties of RPC, including ultimate strength, Young's modulus, elastic response, ductility and capacity to absorb energy, etc, are better than those of HPC, SFRC and NSC. It is considered to be a 
revolutionary material that provides a unique combination of ductility, strength, durability, and aesthetic flexibility.

\section{Concept of RPC-BSS}

To take full advantages of RPC and the mechanics of conventional BSS, a new kind of BSS with a box-section rigid member made of RPC is formed and named RPC-BSS. In RPC-BSS, the cable is prestressed to provide part of rigidity of the structure and bear the bracing bars, which act as elastic supports to offer resistance to deformation of the RPC member. The RPC member is mainly subjected to axial loads, at the same time subjected to some bending moment. The deformation of the structure could be controlled by adjusting the degree of prestress, and the value of tensile stress within the RPC members could be controlled too.

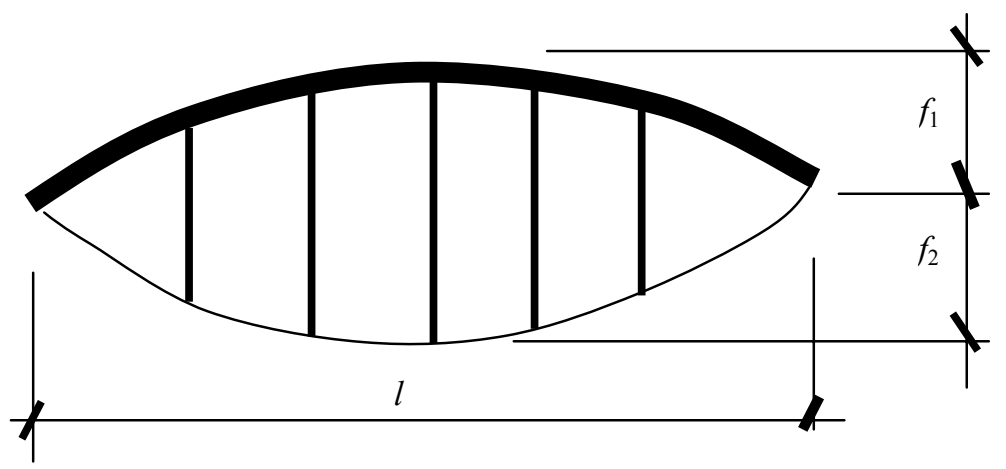

Figure 1: $\quad$ Model of RPC-BSS.

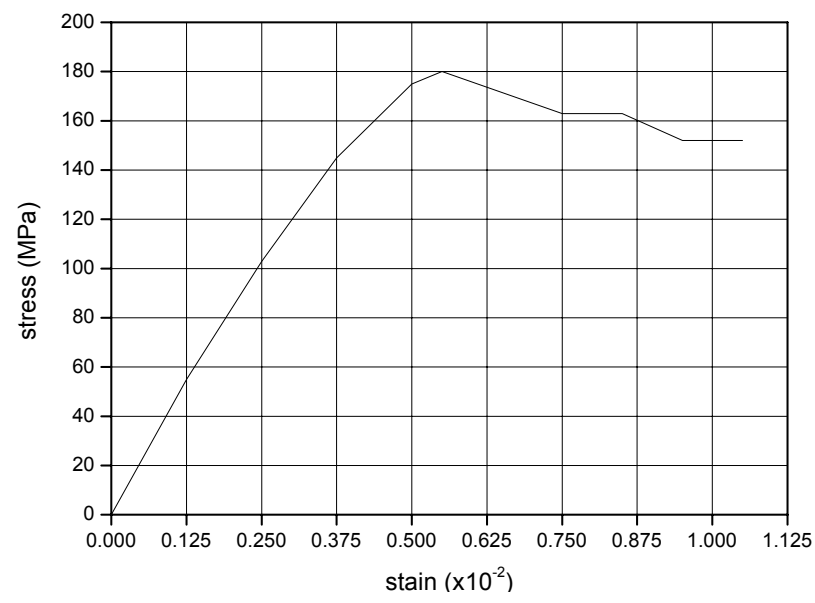

Figure 2: $\quad$ Strain-stress curve of RPC. 


\section{Static properties of RPC-BSS}

During the whole process analysis, the finite element model of RPC-BSS is set up, in which beam elements and link elements are used to model the rigid upper chord, bracing bars and cables, as shown in Figure1. With the software ANSYS, displacement, stress, etc, are calculated and discussed. The curve of strain-stress of RPC [2] used in this calculation is shown in Figure 2.

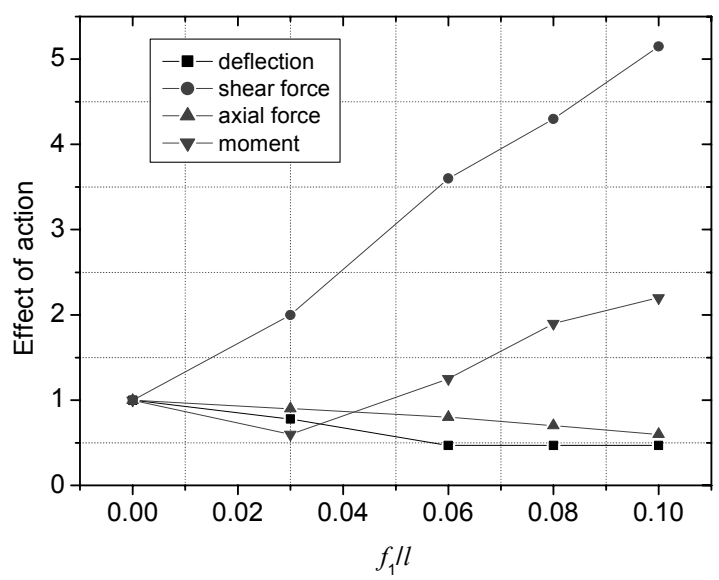

Figure 3: Effect of action versus $\mathrm{fl} / \mathrm{l}$ curve.

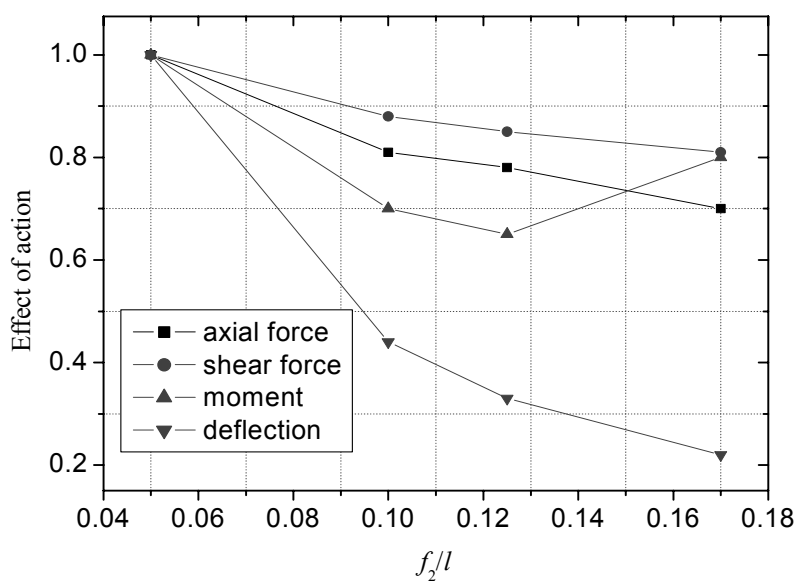

Figure 4: $\quad$ Effect of action versus $\mathrm{f} / \mathrm{l}$ curve.

In static analysis, the influence of different factors are taken into consideration, such as high-to-span ratio, tag-to-span ratio, degree of prestress in tendon, and so on. The diagrams of deflection and internal force induced by the uniform distributed load which is applied along the half-span are shown in Figures 3, 4 and 5. 
Figure 3 shows that 0.06 is a reasonable high-to-span ratio because the structure provides high rigidity and less internal force. Figure 4 shows that 0.125 is a reasonable tag-to-span ratio because of the same reason. Figure 5 shows that the maximum deflection increases first and then decreases as prestress increases, while moment varies inversely. The maximum axial force and shear force increase, and it is found that these curves vary slowly.

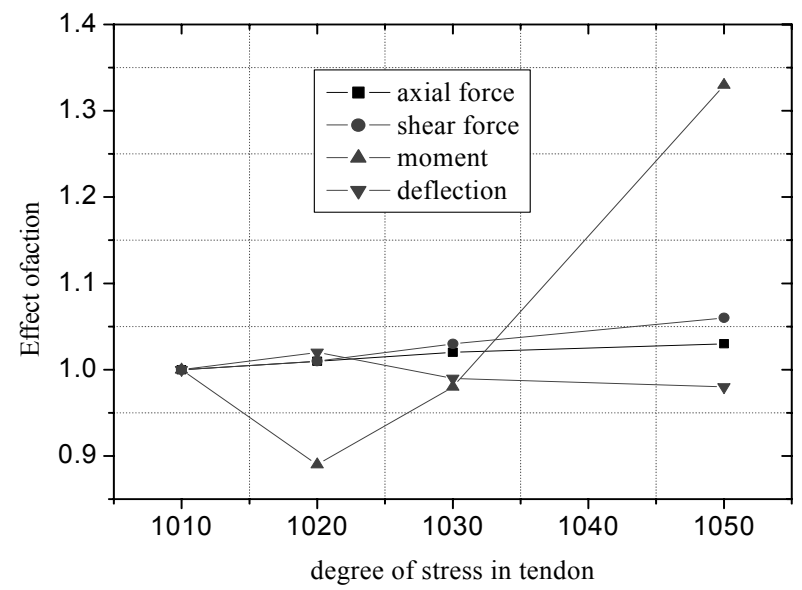

Figure 5: $\quad$ Effect of action versus stress in tendon curve.

\section{Dynamic properties of RPC-BSS}

With the software ANSYS, the modal analysis of RPC-BSS is carried out and the main vibration frequencies are developed, as shown in Figure 6.

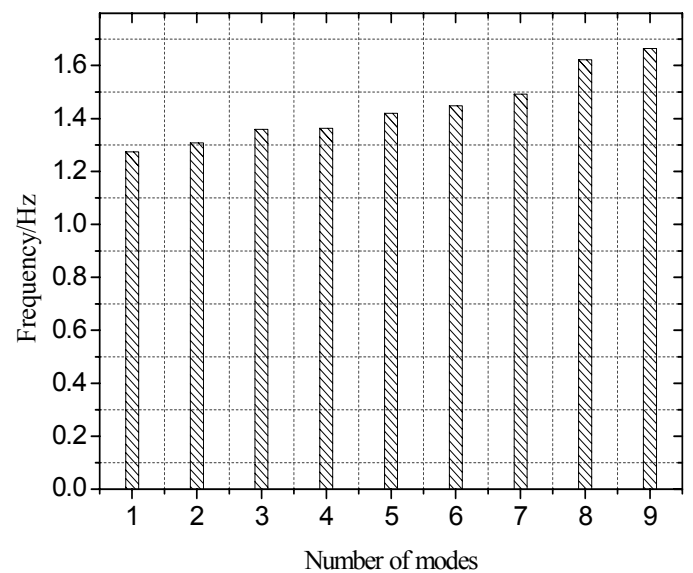

Figure 6: Natural frequency components.

Figure 6 shows that the natural frequencies are densely distributed. And the curve varies slowly. 


\section{Conclusions}

0.06 is a reasonable high-to-span ratio and 0.125 is a reasonable tag-to-span ratio because of the point at which the structure provides high rigidity and induces less internal force. The natural frequencies are densely distributed. RPC-BSS is a type of practicable structure from the point of view of safety, durability, economy, fabrication etc. There are also some problems that are needs to be solved in the future about RPC-BSS, for example, reasonable cross-section, detail design, earthquake resistant behaviour.

\section{References}

[1] BAI Z.X. and LIU X.L., A new kind of space structure-beam string structure. Space Structure, 7(2), pp.33-38, 2001.

[2] GAO R. and Jiang L.C., Experimental report on RPC beam structure, 2008, Beijing Jiaotong University, Beijing, P.R. China. 